\title{
A HYBRID MANUFACTURING APPROACH FOR LOW VOLUME HIGH TEMPERATURE THERMOPLASTIC / THERMOSET MATERIAL MOLDS
}

\author{
Hamed Kalami \\ Mechanical Automotive, and Materials Engineering \\ University of Windsor \\ Windsor, Canada \\ kalami@uwindsor.ca
}

\author{
R. Jill Urbanic \\ Mechanical Automotive, and Materials Engineering \\ University of Windsor \\ Windsor, Canada \\ jurbanic@uwindsor.ca
}

\begin{abstract}
The mold costs for low volume production molds can be expensive due to the mold material, the process planning time, and the fabrication costs. The focus of this research is to develop a methodology to fabricate molds for low volume production, where the production quantities vary between $1-200$ components. For this application, the cycle time is not an issue. Employing an additive manufacturing solution could reduce the required amount of materials and the processing planning costs, but there are cost, or technology feasibility issues related to constructing a mold directly from a CAD file. Consequently, a hybrid manufacturing approach is taken where an AM process (material extrusion based) is used to create a sacrificial pattern for specialty, low cost, interchangeable inserts manufactured using an epoxy (Aremco 805). An over molding case study is carried out using a high temperature molding material - Technomelt-PA 7846 black. The pattern, insert, coating, and mold fabrication is discussed, as well as the initial results. The initial material cost estimates to fabricate this over molding solution is approximately $\$ 140$ US. The durability of the RTV coating and the inserts needs to be determined to yield the final solution costs.
\end{abstract}

Keywords-mold fabrication; low volume; high temperature plastic materials; additive manufacturing

\section{INTRODUCTION}

The competitive landscape in the manufacturing domain is increasing in our global economy. The plastic mold making industry, which is a multi-million industry consisting of mainly small and medium size enterprises (502 establishments with 5,300 employees and $\$ 926$ million in shipments [1]), is actively pursuing opportunities to reduce tooling costs and processing time. The design solutions depend on the production volumes and planning horizons, and different mold materials and fabrication strategies are required for low volume, medium volume, and high-volume production. The focus of this research is on low volume production, where the production quantities vary between $1-200$ components, the cycle time is not an issue, and the target application is 'over molding' for specialty applications, or prototype low volume production.
Low volume production molds are utilized in many industrial sectors, and can be used to test product functionality, or employed to create sample components for assembly automation 'test and tune' applications for automotive high production systems. 'Soft' tooling or temporary molds are fabricated to produce a limited number of products. If the design is not optimal, another mold can be fabricated for the updated design. The over molding process involves the use of two separate materials to form one cohesive component. The most common type of over molding is insert molding. Insert over molding is an injection molding process where one material is molded "over" a secondary "substrate" material.

Low volume molds are less costly than medium or high volume molds, but the material, process planning, and fabrication costs may still be thousands of dollars. Additive Manufacturing (AM) approaches have the potential to reduce process planning time, and to reduce the both the time and manpower associated with the mold fabrication process.

There are several AM processes that have been developed: vat photopolymerization, binder jetting, material extrusion, powder bed fusion, directed energy deposition, material jetting, and sheet lamination. The AM process family is capable of producing complex geometries without any in-depth process planning, and are used in the automotive, aerospace domains, as well as in the medical and dental domains [2]. The common element to all these processes is that a three-dimensional (3D) part is developed from layering two dimensional cross sections successively to create the final solid. Undercuts, free form geometry, and blind features are manufactured "easily", especially compared to traditional machining processes. Presently, there are limited choices of material available for AM processes and anisotropic properties are exhibited due to the material bonding [3], [4]. There are compatibility issues with respect to the AM build process material with the molding material, and also limitations with respect to their cost effectiveness for 'lower' to intermediate production volume quantities as the product fabrication times may be long. Therefore, a rapid tooling design and fabrication strategy needs to be developed [5], which is the focus of this research. There are two solution paths being proposed: (i) employing an AM process to fabricate a mold (with the addition of a coating if 
there are material compatibility issues), and (ii) employing hybrid mold fabrication strategies.

The goal of this research is to develop solutions to fabricate a $200 \mathrm{~mm}$ x $150 \mathrm{~mm}$ x $50 \mathrm{~mm}$ core and cavity mold set that can produce up to 200 parts for $\leq \$ 1000$. In this study, the molding material is Technomelt-PA 7846 black which is a polyamide plastic. Its mechanical properties are shown in Table I. Technomelt is a high melting temperature polyamide (application temperature: 200 to $240{ }^{\circ} \mathrm{C}$ [6]), which limits the usable mold materials.

TABLE I. PROPERTIES OF TECHNOMELT-PA 7846 BLACK

\begin{tabular}{|l|l|}
\hline \multicolumn{1}{|c|}{ Mechanical property } & \multicolumn{1}{c|}{ Value } \\
\hline Density, $\mathrm{g} / \mathrm{cm}^{3}$ & 0.98 \\
\hline Melting Viscosity at $230{ }^{\circ} \mathrm{C}, \mathrm{mPas}$ & 3,000 \\
\hline Glass Transition, ${ }^{\circ} \mathrm{C}$ & -30 \\
\hline Working Temperature, ${ }^{\circ} \mathrm{C}$ & -40 to 130 \\
\hline Softening point, ${ }^{\circ} \mathrm{C}$ & 170 to 180 \\
\hline
\end{tabular}

\section{LITERATURE REVIEW}

Rodet et al. used the Stereoligthography (SLA) [7] process to fabricate molds using the DSM Somos 7110 resin and SL 7510 materials, and SLA-250 and SLA-3500 machines. Variable layer thicknesses were explored and correlated to a surface roughness. The SLA tools had limitations due to their low thermal and mechanical properties $\left(60-70^{\circ} \mathrm{C}\right.$ - little strength left). Hopkinson et al. utilized an epoxy resin SL-5170 with a SLA-500 machine. The operating conditions were at a higher temperature $\left(185^{\circ} \mathrm{C}\right)$. It was necessary to use different processing parameters for the molding compared to an aluminum mold solution, and there was difficulty ejecting the components (polypropylene material). Kovács et al. analyzed the warpage issues related to mold thermal conductivity, and explored using an epoxy resin filled with metal powder to increase stiffness and heat conductivity [8]. The SLA process family was not feasible for directly fabricating a mold set for components to be molded with the Technomelt-PA 7846 black material at this time.

The binder jetting process is an additive manufacturing process that fabricates components and assemblies by layering powered material and applying a binder where a 'solid interior' should be. The binder jetting family of technologies is capable of printing variety of powder materials, which includes polymers, metals, ceramics, sand, and plasters. However, the raw material costs for metals [9] are high compared to the raw stock costs used for machining; consequently, this process may be more suited to a medium volume production application. This is also true for the powder bed fusion processes.

The material extrusion family for AM processes consists of placing individual beads side by side to build up a layer. A contour boundary curve is extruded, and a raster fill strategy is used to fill the appropriate interior regions. The material is extruded through a nozzle. Typically, the build material is a thermoplastic such as Acrylonitrile Butadiene Styrene (ABS) or Polylactic Acid (PLA), although a polycarbonate can be employed. Both this process and the readily available material selections are problematic. Fabrication issues exist with the surface finish, anisotropic mechanical properties, and voids within the tool paths [10], but these issues are secondary. The glass transition temperature for ABS is approximately $105^{\circ} \mathrm{C}$, and the melting point for PLA 150 to $160{ }^{\circ} \mathrm{C}$. Polycarbonate has a glass transition temperature at approximately $147^{\circ} \mathrm{C}$. The softening point for the Technomelt-PA 7846 black is 170 to $180^{\circ}$. Thus, the material extrusion processes do not lend themselves to a high temperature mold fabrication application.

Consequently, directly printing a mold set from a Computer Aided Design (CAD) model is not feasible due to cost issues (binder jetting and powder bed fusion), or due to the available build materials (SLA and material extrusion). Therefore, a 'rapid tooling' hybrid mold manufacturing approach is to be explored.

S. Ma et al. investigated the feasibility of making reinforced epoxy molds. The metal, ceramic and mineral powders were mixed with epoxy to increase its mechanical properties. Different ratios of the powders were studied to find the best mixture to optimize mechanical and thermal properties. $20 \%$ of alumina powder found to be the most suitable additive [11]. T. Tabi et al compared thermal, mechanical and thermomechanical properties of injection molding of PLA material into epoxy-based PolyJet and conventional steel (P20) mold. They found that it is possible to mimic the thermomechanical properties of nucleated PLA which is injected into hot steel mold by injecting it into an epoxy-based PolyJet mold [12]. Therefore, using a metallic-resin mix for controlling the heat transfer and providing structural integrity is a valid solution approach for a mold, and is a focus of the solution presented here.

\section{METHODOLOGY}

For the hybrid manufacturing strategy, a disposable pattern for the core and cavity mold components will be manufactured using an AM process (rapid tooling), and the balance of the components are machined or cast to create permanent and interchangeable components. Two hybrid options are explored: (i) create a shell of each core and cavity mold surfaces, and back fill the shell with a filler material, and (ii) create a pattern, and use the pattern to create an interchangeable insert with a suitable resin material. For this paper, option 2 is explained. The process flow is presented in Fig. 1.

\section{A. Mold Design}

The mold base consists of two aluminum blocks sized to accommodate a variety of inserts. There is a $35 \mathrm{~mm}$ border around the machined rectangular cavities that are to be used for the mold inserts. Fig. 2 shows the mold base blocks and its sections, where: the A features ( 2 per side) are entrance holes specifically designed for the epoxy filling operation; the $\mathrm{B}$ features (4 per side) are to allow the users to quickly interchange epoxy inserts for new production runs; the $\mathrm{C}$ feature is the insert cavity, which includes a $30^{\circ} \mathrm{draft}$ angle and $15 \mathrm{~mm}$ corner fillet radii for ease of interchangeably; and the D features ( 2 per side) are used to align the core and cavity blocks. These D features are also used to align the match plates to the mold set. The ejector features are threaded to fix the insert into position, and work as an ejector mechanism that pushes the used insert out of mold base cavities. The epoxy 
insert contains nuts for stable location. To reduce bonding of the epoxy material to the aluminum block, an RTV silicone material (Mold Star® 30 [13]) is applied to the cavity surface before pouring the epoxy.

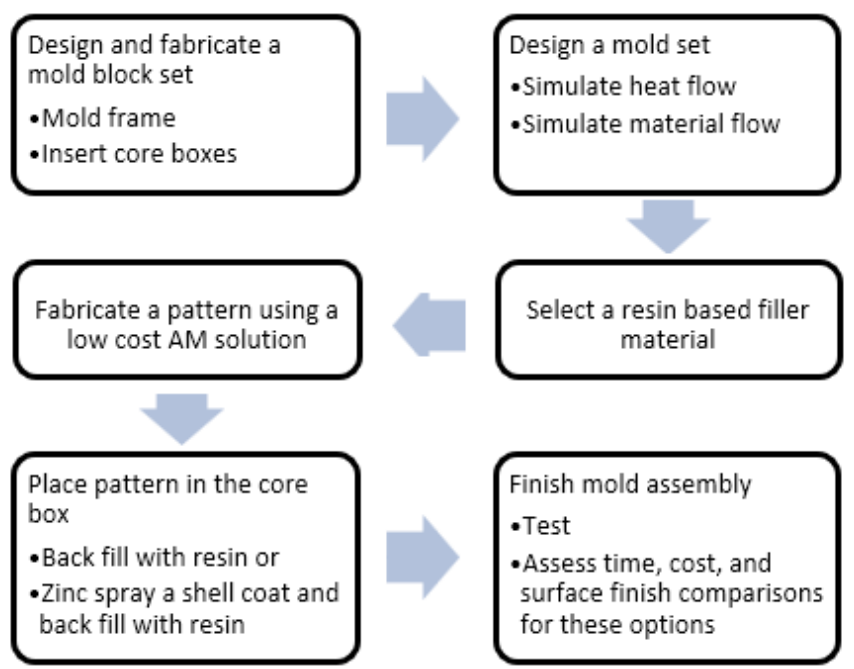

Figure 1. Hybrid mold design process flow.

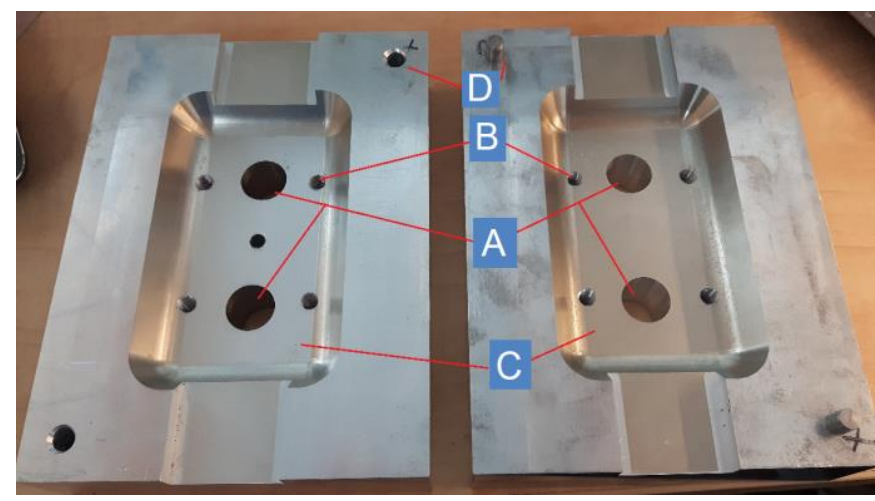

Figure 2. The mold bases- (A) the holes that epoxy is poured into the epoxy cavities (B) the ejector bolt of the epoxy block (C) epoxy filled cavities (D) hole-pin locators.

\section{B. Mold Making Process}

To fabricate the molding cavities, a pattern is needed. As the pattern may have shape complexity, but does not need to have strength, low cost additive manufacturing processes and materials are ideal for this 'rapid tooling' application. Pattern design characteristics will vary depending on the final product requirements. The parting line and features will be unique for a general product as compared to an over molding solution. Once the sample is divided from the parting line, each segment is attached to each side of a match plate. The locating pins (feature D) of the mold base align pattern, the match plate, and the mold bases. Due to space constraints, the analyses for the various epoxy materials are not included. However, from the experimental activities, is was found that the Aremco 805 (Table II) had the low shrinkage, viscosity, mechanical properties at room temperature and $200^{\circ} \mathrm{C}$, the temperature resistance, and the desired heat transfer characteristics.
TABLE II. PROPERTIES OF AREMCO 805 EPOXY [14]

\begin{tabular}{|l|l|}
\hline \multicolumn{1}{|c|}{ Mechanical property } & \multicolumn{1}{c|}{ Value } \\
\hline Thermal Conductivity $(\mathrm{W} / \mathrm{m} 2 . \mathrm{K})$ & 70.98 \\
\hline Tensile shear $(\mathrm{Pa})$ & $1.2 \mathrm{e}+7$ \\
\hline Flexural strengths $(\mathrm{Pa})$ & $1.06 \mathrm{e}+8$ \\
\hline Linear shrinkage $(\mathrm{m} / \mathrm{m})$ & 0.003 \\
\hline Shore D Hardness & 87 \\
\hline
\end{tabular}

In Fig. 3 (a) the build layers for the pattern are shown as visualized in the Insight ${ }^{\circledR}$ processing planning software (used for fused deposition modelling machines [15], in Fig. 3 (b) the original built pattern and an RTV coated pattern show the different surface textures, and in Fig. 3 (c), an over molding pattern is displayed, where the pattern includes representative over molding elements, the over molded region, and the match plate. RTV silicone rubber is used to coat the pattern to smooth the surface and to provide an easy part release. Surface roughness is an issue with the AM process family and researchers engaged in applying AM solutions for mold manufacturing have had ejection issues connected with the build surface finish. Post processing operations are typically required if a smooth surface is desired. Here, a material extrusion process and $\mathrm{ABS}$ material is employed to create the pattern, and in lieu of chemical smoothing or finish sanding, the RTV is employed to smooth the surface and be a releasant.

The patterns were easily removed from the epoxy. The final inserts are placed in the mold halves, as shown in Fig. 4. The over molded components have pockets for their placement in the insert. Additional end seals are also fabricated from the AM pattern for flash control.

The interior cavity surfaces are smooth. The process planning for the pattern was less than 1 minute, the assembly time for the pattern and match plate was approximately $1 / 2$ hour, and the cure time for the epoxy and the silicone was approximately $26 \mathrm{hrs}$. The time where an employee directly interacts with the fabrication process is short, and the required skill level for this insert solution is very low.

\section{Injection molding analysis}

The injection parameters need to be determined for the injection molding operation. Here, Autodesk Mold Flow Advisor 2017 is applied to determine the fill time, and the injection pressure using the input data provided in Table III.

TABLE III. MOLD FLOW SIMULATION INPUT DATA

\begin{tabular}{|l|l|}
\hline \multicolumn{1}{|c|}{ Injection property } & \multicolumn{1}{c|}{ Value } \\
\hline Injection Temperature $\left({ }^{\circ} \mathrm{C}\right)$ & 225 \\
\hline Mold Temperature $\left({ }^{\circ} \mathrm{C}\right)$ & 40 \\
\hline Gate Diameter $(\mathrm{mm})$ & 3 \\
\hline Maximum machine injection pressure $(\mathrm{MPa})$ & 1 \\
\hline
\end{tabular}

As shown in Fig. 4, the material injection gate is centrally located; consequently, the maximum fill time is 0.8 seconds along the longitudinal axis, but there are variants in the fill time, as shown in Fig. 5a. The temperature contours and cooling times are dependent on the system configuration, and are not presented here. The maximum injection pressure is at 
the gateway, and $0.11 \mathrm{MPa}$. The average injection pressure is very low and is almost homogenized (Fig. 5b).

(a)

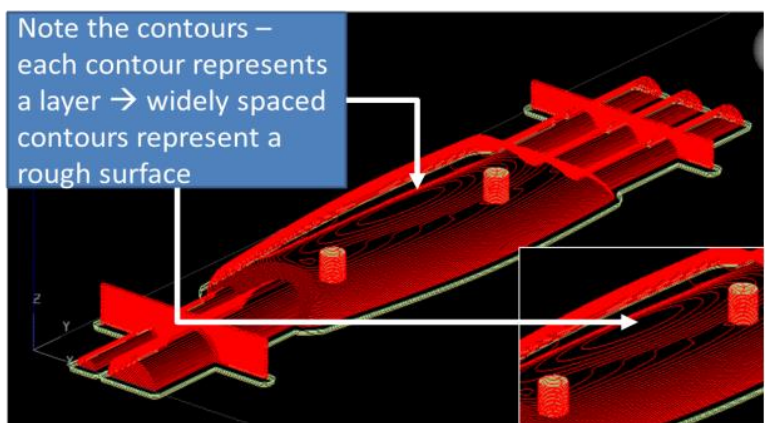

(b)
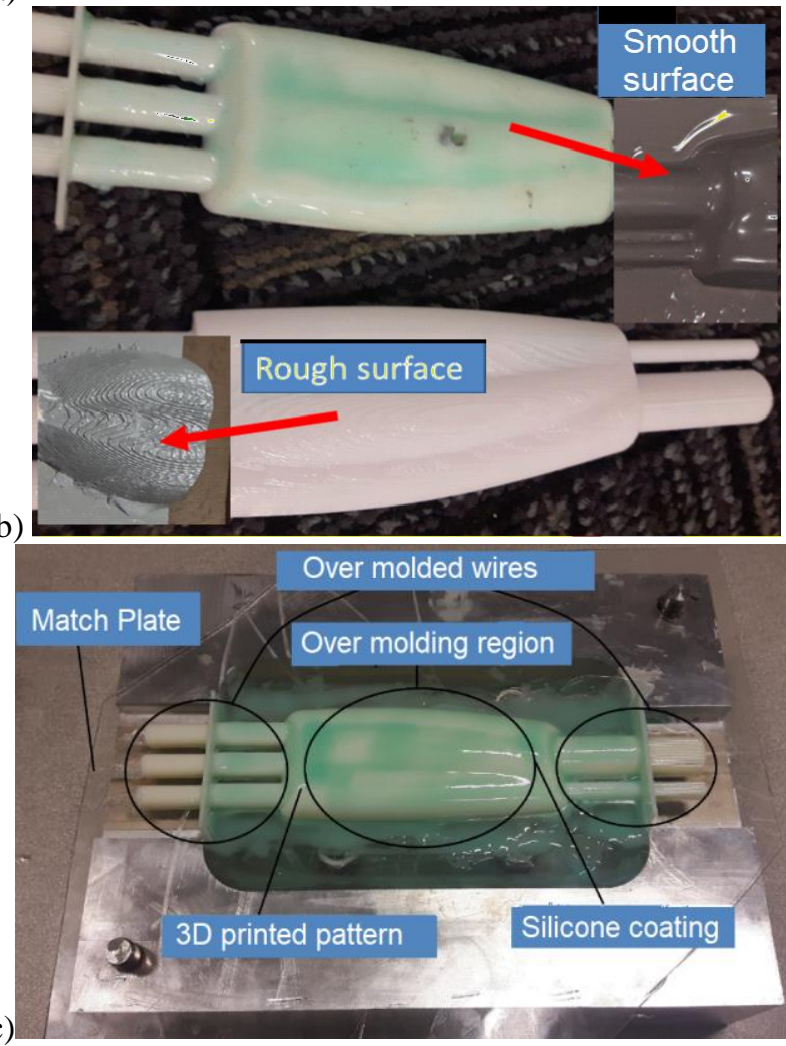

Figure 3. (a) The build layers as reprsented by the Insight ${ }^{\circledR}$ process planning software, (b) the built and coated patterns, and (c) The parts of the epoxy mold making process

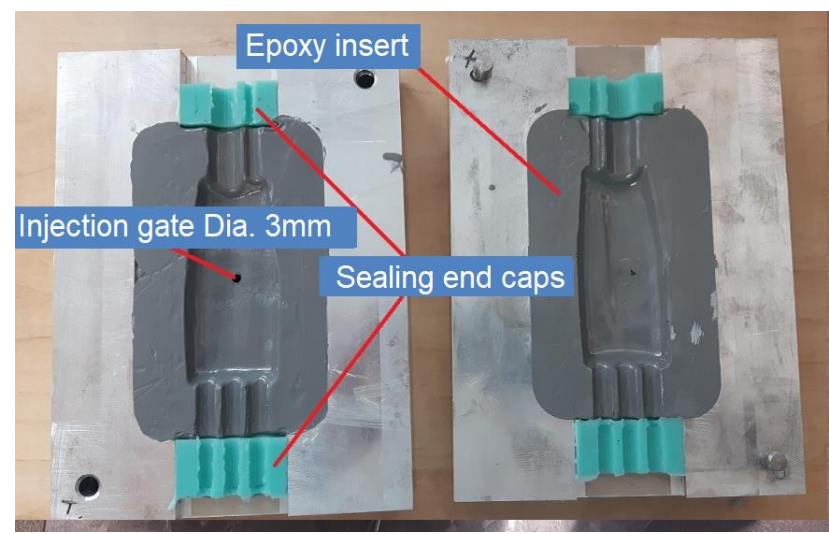

Figure 4. Epoxy inserts, and sealing end caps for the mold set.

\section{EXPERIMENTAL RESULTS}

In order to test the mold, the test part (Fig. 6) was placed inside the mold. The over molding region is encircled in the picture. The wires fit into the sealing end caps. The injected Technomelt-PA 7846 black covered the wire splice completely, and as shown in Fig. 7, the over molded product has a very smooth surface. The resulting product did not adhere to the mold cavity surface, and was removed easily. The material costs for this solution are divided into 2 categories: capital costs and consumable cost. (Table IV). The overall cost is approximately $\$ 140 \mathrm{US}$, which is much less than the cost of making a permanent aluminum mold using conventional machining processes (estimated to be $\$ 840$ US, using a 3 axis mill, and a final polishing operation, Table V).

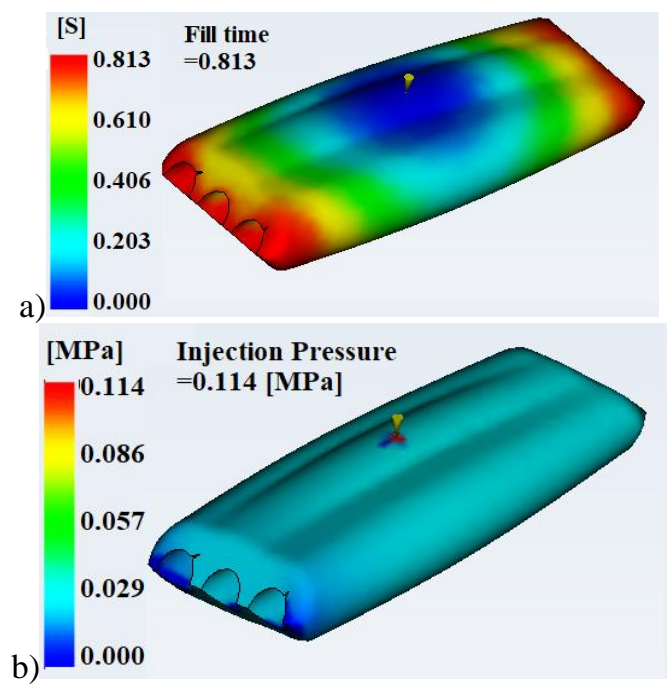

Figure 5. a)Fill time contours. B) Injection pressure contours.

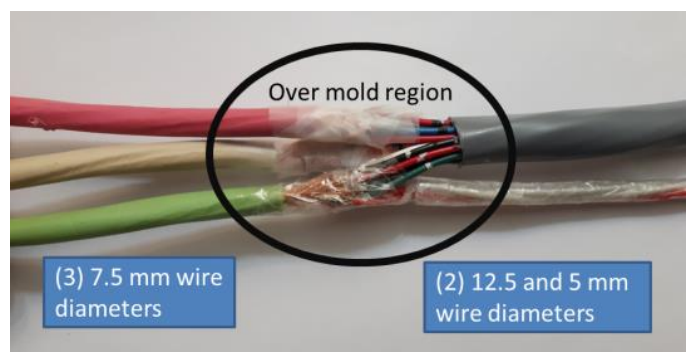

Figure 6. Over molding test application.

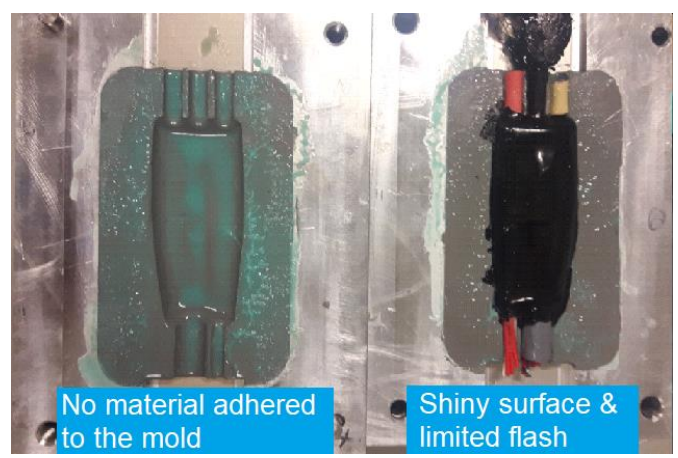

Figure 7. The over molded product in the mold. Note that there is no residual material in the left cavity and minimal flash in the right cavity. 
For machining a permanent aluminum mold, the Mastercam verification analysis indicates that 4 hours machining is required to fabricate the two mold blocks (Fig. 8). The machining process includes a face milling, two rough machining operations, followed by a finishing pass with a large step over. In Table V, a detailed cost breakdown is presented.

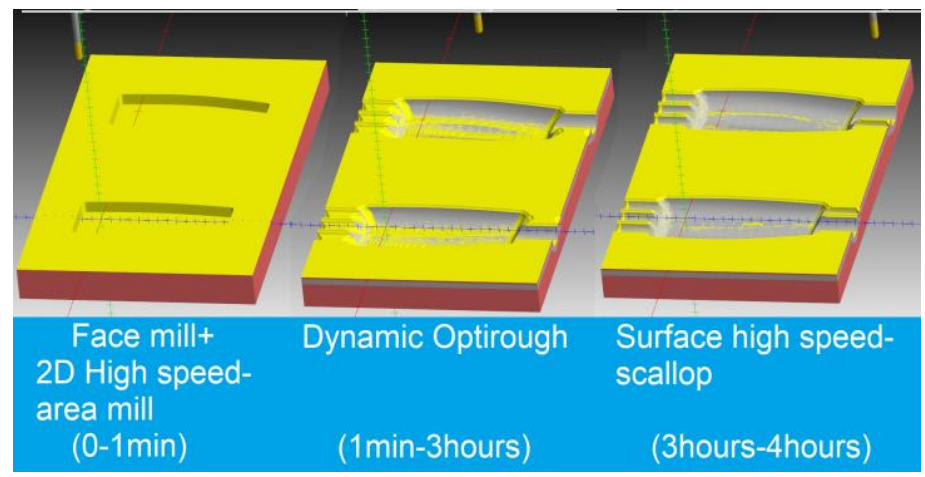

Figure 8. Permanent mold machining strategies and verification model.

TABLE IV. MATERIAL COSTS FOR FABRICATING THE EPOXY MOLD

\begin{tabular}{|l|l|c|}
\hline \multirow{2}{*}{ Capital costs } & Aluminum blocks & $\$ 70$ \\
\cline { 2 - 3 } & Match plate material & $\$ 5$ \\
\hline \multirow{3}{*}{ Consumables } & AM pattern printing & $\$ 16$ \\
\cline { 2 - 3 } & Aremco 805 & $\$ 44$ \\
\cline { 2 - 3 } & RTV Silicone & $\$ 6$ \\
\hline Total (US Dollar) & & $\mathbf{\$ 1 4 1}$ \\
\hline
\end{tabular}

TABLE V. COSTS FOR MACHINING A PERMANENT MOLD (NOTE: THIS DOES NOT INCLUDE MATERIAL COSTS)

\begin{tabular}{|l|c|c|}
\hline \multicolumn{1}{|c|}{ Process } & Time & Cost \\
\hline Process planning & 6 hours & $\$ 300$ \\
\hline Set up time & 1 hour & $\$ 80$ \\
\hline Machine run & 4 hours & $\$ 400$ \\
\hline Polishing & 16.2 inch $^{2}-2 \mu \mathrm{m}$ Rough & $\$ 60[16]$ \\
\hline Total (US Dollar) & & $\$ \mathbf{8 4 0}$ \\
\hline
\end{tabular}

The final product is shown in Fig. 9. There is some flash; consequently, the sealing end cap solution will be redesigned.

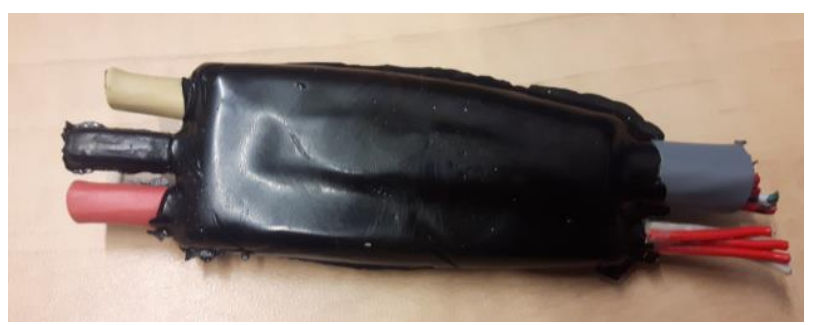

Figure 9. Final over molded product. The production quantity limitation for this mold solution will be caused by the wear of epoxy insert edges, the durability of the epoxy material from thermal shocks, and the durability of RTV silicone coating. Future experiments will be performed to determine the mold tool life.

Although the durability is important, epoxy insert replicates for a specific product will not increase the overall cost significantly. Therefore, if there are tool life issues, the RTV silicone coating can be applied as needed, and several epoxy inserts can be produced with the AM pattern.

\section{SUMMARY AND CONCLUSIONS}

A lower cost mold fabrication solution is presented for low volume, high temperature molding materials. AM processes cannot directly be used to fabrication a low-cost solution, but AM processes can be utilized to manufacture a sacrificial intermediary pattern. This pattern is employed to create an insert (or inserts - as required) made from an epoxy. Further experiments need to be performed to determine the tool life. Once this is completed, a comprehensive cost analysis can be developed to determine the overall costs of this build strategy.

\section{ACKNOWLEDGMENTS}

This research is partially funded by the NSERC Engage Grant, and the Ontario Centers of Excellent VIP programs. Special thanks to the industrial partner for their support.

\section{REFERENCES}

[1] Government of Canada, Plastics machinery and moulds https://www.ic.gc.ca/eic/site/plastics-plastiques.nsf/eng/p101409.html, 2012, [Last cited on Feb 2018].

[2] T. T. Wohlers, "Wohlers report, State of the industry, annual worldwide progress report, Wohlers Associates," 2017.

[3] C. O. Balderrama-Armendariz, E. MacDonald, D. Espalin, D. Cortes-Saenz, R Wicker, A. MaldonadoMacias, "Torsion analysis of the anisotropic behavior of FDM technology" The International Journal of Advanced Manufacturing Technology, Springer-Verlag London Ltd., part of Springer Nature, pp. 1-11, 2018.

[4] A. Bellini, S. Güçeri, "Mechanical characterization of parts fabricated using fused deposition modeling" Rapid Prototyping Journal, vol. 9, Issue. 4, pp. 252-264, 2003.

[5] P. Dunne, S.P. Soe, G. Byrne, A. Venus, A.R. Wheatley, "Some demands on rapid prototypes used as master patterns in rapid tooling for injection moulding", Journal of Materials Processing Technology, vol. 150, pp. 201-207, 2004.

[6] TECHNOMELT PA 646 BLACK, http://na.henkel-adhesives.com/product-search1554.htm?nodeid=8797758029825, 2018, [Last cited on Feb 2018].

[7] V. Rodet, J. S. Colton, "Properties of rapid prototype injection mold tooling materials" Polymer Engineering \& Science, vol. 43, Issue. 1, pp. 125-138, 2003.

[8] J. G. Kovács, F. Szabó, N. K. Kovács, A. Suplicz, B. Zink, T. Tábi, H. Hargitai, "Thermal simulations and measurements for rapid tool inserts in injection molding applications", Applied Thermal Engineering, Vol. 85, pp. 44-51,2015.

[9] ExOne, http://www.exone.com/Resources/Materials, 2018, [Last cited on Feb 2018]

[10] S-H. Ahn, M. Montero, D. Odell, S. Roundy, P. K. Wright, "Anisotropic Material Properties of Fused Deposition Modeling ABS" Rapid Prototyping Journal, vol. 8, issue. 4, pp.248-257, 2002.

[11] S. Ma, I. Gibson, G. Balaji, and Q.J.Hu "Development of epoxy matrix composites for rapid tooling applications," Journal of Materials Processing Technology, vol. 192-193, pp. 75-82, 2007.

[12] T. Tabi, N. K. Kovacs, I. E. Sajo, T. Czigany, S. Hajba, "Comparison of thermal, mechanical and thermomechanical properties of poly(lactic acid) injection-molded into epoxy-based Rapid Prototyped (PolyJet) and conventional steel mold" Journal of Thermal Analysis and Calorimetry Vol. 123, pp. 123: 349, 2016.

[13] Mold Star 30, https://www.smooth-on.com/products/mold-star-30/, Smooth on 2018, [Last cited on Feb 2018].

[14] Aremco-Bond 805, https://www.aremco.com/news-item/aremco-bond-805-hightemp-thermally-conductive-epoxy-now-available/, 2015 [Last cited on Feb 2018].

[15] Insigt Software, http://www.smg3d.co.uk/3d_design_software/insight_software, SMG 3D 2018, [Last cited on Feb 2018].

[16] K. J. Fisher, M. J. Lobaugh, R. M. Michael, S. K. Sweeney, P. J. Kuvshinikov, "Development of a cost model for manual tool polishing", The International Journal of Modern Engineering Vol. 6, 2006 